\title{
Game-theoretic analysis of opportunistic spectrum sharing with imperfect sensing
}

\author{
Fang Wang ${ }^{1}$, Jinting Wang ${ }^{1 *}$ and Wei Wayne $\mathrm{Li}^{2}$
}

\begin{abstract}
We consider the strategic behavior of secondary users (SUs) in a cognitive radio system where SUs opportunistically share a single primary user (PU) band over a coverage area. The service of an SU can be interrupted by a PU in a preemptive manner, and the interrupted SU may abandon the system or wait until the PU band is sensed available. In the latter case, if spectrum sensing errors occur, they will cause misdetections and false alarms which impact the system's performance heavily. In this paper, we model this problem as a retrial queueing system with server breakdowns and recoveries in which the interrupted SUs are treated as retrial customers. They will retry for using the PU band after some period of time due to interruptions or misdetections. The arrival of a PU during service of an SU is modeled as a server breakdown, and the recovery time is equivalent to the service time of this PU. We focus on the behavior of arriving SUs who can make decisions on whether to join the system or to balk based on a natural cost structure and the delays caused by PUs' interruptions, which can be studied as a non-cooperative game. The equilibrium and optimal strategies of SUs are both derived. Furthermore, to bridge the gap between the individually and socially optimal strategies, a novel strategy of imposing an admission fee on SUs to join the retrial group is proposed. Finally, some numerical examples are presented to show the effect of several key parameters on the system performance.
\end{abstract}

Keywords: Cognitive radio, Imperfect spectrum sensing, Retrial queue, Strategic behavior, Admission fee

\section{Introduction}

Spectrum is recognized as one of the limited transmission resources which face the challenges of the ever increasing demand for higher data rates and lower latency in communication networks. Cognitive radio (CR) first introduced by Mitola [1] can alter its transmitter parameters to accommodate the environment where it operates to utilize spectrums more efficiently. The potential of cognitive radio is being recognized not only by the military but also by the commercial sector, for example, in intelligent transportation, in cellular communications, and in public safety.

Previous studies have shown that the utility of the spectrum is very low under conventional static spectrum access strategies [2]. As the users' demands increase while the amount of dedicated spectrum is limited, more and more network users have to choose dynamic spectrum

\footnotetext{
*Correspondence: jtwang@bjtu.edu.cn

1 Department of Mathematics, Beijing Jiaotong University, No. 3 Shang Yuan Cun, Beijing, China

Full list of author information is available at the end of the article
}

access (DSA) which has been considered as a viable solution to alleviate this spectrum scarcity and improve radio communication efficiency. An amount of work focused on the performance analysis of various systems; however, they neglect the competition between different users. The relationship between users and operators of radio networks is also worth considering.

There are two kinds of users in cognitive radio networks, namely, licensed (primary) users and unlicensed (secondary) users. The secondary users can use the capabilities of spectrum sensing, learning, and adaptation to use the licensed spectrum to transmit, thereby enabling coexistence and leading to higher overall spectral efficiency. In general, due to the fact that in CR networks primary users (PUs) have priorities over secondary users (SUs) and the arrivals of PUs will interrupt the service of SUs (if any), researchers use queueing systems with breakdowns to characterize the interruption process involved. To SUs, the preemptive priority scheme underlying the 
CR network implies that the arrival and service of any PU will bring a breakdown and repair process by means of queueing way of saying.

In CR systems, in most cases, there is no centralized controller to regulate channel access. Hence, a rational secondary user needs to give his strategies relying on local information and has to adapt to the environment quickly. It is natural to form a spectrum market including cooperation, pricing, and leasing since PUs have a limited number of spectrum bands. In these spectrum markets, user behaviors can be modeled and analyzed by economic games. Tran et al. [3] studied delay-sensitive secondary users via pricing strategies in a dynamic spectrum market with a single PU band. Do et al. [4] considered a duopoly market with cooperative and non-cooperative models and provided the analysis of the pricing effect on equilibrium behavior of SUs by using the $M / G / 1$ queue with breakdown.

Game-theoretic spectrum sharing criteria could be used to maximize both primary and secondary users' satisfaction (see [5-7]). Several studies in the literature [8-11] considered the decentralized behavior of SUs and adopted queueing-game approach to investigate the interactions between PUs and SUs in the CR networks. Li and Han [8] studied the discrete model in a more applicable way which obtained the threshold of queue length to characterize the optimal joining strategy of SUs. Do et al. [9] investigated the socially optimal strategy of SUs in unobserved queueing system in cognitive radio base station. Jagannathan et al. [10] illustrated SUs utilizing white spaces that were not used by PUs in the unobservable case by the same model but did not consider the optimization strategy. All of these works [8-10] assumed that there is a queue in front of the PU band and the new arrivals of SUs will enter the queue according to a firstin-first-out (FIFO) discipline. Recently, Wang and Li [11] considered the strategic behavior of SUs with retrials but assumed that the interrupted SU did not leave the service area and it would get service immediately once the occupied PU completed service and left the band. It should be noted that, in all the aforementioned papers, spectrum sensing is assumed to be perfect. None of these works considered sensing failure problem although sensing failures occur in practice and have non-ignorable impact on the system.

Indeed, spectrum sensing plays a vital role in CR networks due to unreliability of wireless channels and users' congestions. In principle, spectrum sensing is imperfect. When an idle band is sensed busy, a false alarm is said to occur. A misdetection refers to the situation that a busy band is sensed idle. These two kinds of errors have significant effects on the performance of CR systems. The probability of a false alarm and that of a misdetection should be kept below a certain level to guarantee the
QoS of PUs and also SUs, i.e., the system performance is acceptable. In [12-18], the authors took imperfect sensing into account. Hoang et al. [12] considered a CR system with one single slotted channel sharing by a PU and an $\mathrm{SU}$, and the problem was formulated as a partially observable Markov decision process. It was shown that the optimal control policies could achieve significant performance gain. In [14], a two-dimensional discretetime Markov chain is used to model a multiple-channel CR system with imperfect sensing. In [15], the multiplechannel CR system with unreliable spectrum sensing was discussed and the authors employed a two-dimensional continuous-time Markov chain model to analyze the system. However, strategic behavior of SUs has not been taken into account in these studies and the above models cannot reflect the decentralized behavior of SUs along with the opportunistic sharing operation in practice.

In this paper, we focus on the strategic behavior of SUs in CR networks from a game-theory point of view. More specifically, we consider the general carrier sense multiple access (CSMA) protocol arising from wireless communication networks. The basic idea of this CSMA protocol lies in the fact that packets start transmission only if no transmission is ongoing and "listen before talk" protocol is adopted. That is, every user before attempting any transmission listens whether somebody else is already using the channel, avoiding the possible collision. To characterize these factors, we model the $\mathrm{CR}$ system as a constant retrial queueing system with server breakdowns, where SUs get access to the PU band according to "listen before talk" protocol as retrial customers. The PU band is considered as a server, and the PUs have the higher priority over all SUs. When the PU arrives, it will occupy the PU band immediately no matter whether the band is serving an SU or is in an idle state. Under the assumption of imperfect spectrum sensing, an extensive study of the Nash equilibrium and the socially optimal strategies for all SUs is carried out in this paper. Besides, to use the PU band more efficiently and eliminate the difference between the equilibrium and the socially optimal strategies, a novel approach of imposing an appropriate admission fee for SUs that decide to join the orbit is proposed under sensing failure. In this way, it is feasible to induce individually optimizing SUs to behave in a socially optimal way.

The works of Jagannathan et al. [10] and Wang and Li [11] are closely related to this paper. The differences between this paper and Jagannathan et al. [10] are as follows. (1) Jagannathan et al. [10] did not consider the optimization strategy. (2) It did not consider the sensing failure problem. Compared to Wang and Li [11], this paper assumes that the interrupted SU will leave the service zone and go back to retrial orbit as a head SU in the retrial queue. Therefore, a new arriving SU has a chance to utilize the PU band directly if the PU band is idle upon arrival. 
In the work of Wang and Li [11], they assumed that the interrupted SU would not leave the service area and it will get service immediately once the occupied PU completes his service. As a result, the new arrivals of SUs during this period (the waiting period of interrupted SU in the service area) have to enter the retrial orbit for later attempts. Evidently, this is more realistic in CR networks. To summarize, the contributions of this paper lie in the fact that we study the SUs' joining behavior in CR networks with a single bandwidth under imperfect spectrum sensing along with constant retrial queueing system for the first time.

The paper is organized as follows. Section 2 presents the model descriptions. In Section 3, we derive the average sojourn time for the arriving SUs who decide to enter the cognitive radio base station when they are not informed the system's information with imperfect spectrum sensing. The equilibrium joining probabilities and socially optimal strategies of SUs are derived. An appropriate admission fee is proposed to eliminate the difference between these two strategies. Section 4 illustrates the effect of various performance measures on the system by analytical and numerical comparisons. Finally, in Section 5, we give some conclusions.

\section{System model}

We consider a cognitive radio base station which incorporates a single PU band that is shared by SUs. It means that the PU band can transmit either one PU packet or one SU packet at one time. We regard the PU band as a server. As PUs have high priorities to use the band, an emerging PU should be served immediately no matter whether the band is serving an SU or in an idle state. SUs can opportunistically use the band when it is not occupied.

The primary SUs and PUs arrive to the system according to a Poisson process with rates $\lambda_{s}$ and $\lambda_{p}$, respectively. The service time for SUs (or PUs) follows an exponential distribution with rate $\mu_{s}\left(\right.$ or $\left.\mu_{p}\right)$. If the server is free when an SU arrives, the SU starts service immediately. Otherwise, if the arriving SU finds the server unavailable or an SU in service is squeezed out by an PU, in both cases, the SU will enter an artificial waiting space called "retrial orbit" in order. When the PU band becomes idle, it will be sensed by the first SU in the orbit and the inter-sensing time follows an exponential distribution with parameter $\theta$. The arrival processes of PUs and SUs, service processes of PUs and SUs, sensing process, and retrial process of SUs are mutually independent of each other.

If the spectrum sensing is perfect, the QoS experienced by PUs should not be affected by the SUs. However, in practice, a PU may experience disruptions by the SUs' imperfect sensing. The first case is that if a secondary user searches for the occupied band incorrectly as idle status, collisions will occur. The second kind of disruption to a PU may occur when an ongoing SU transmitting on a given band fails to detect the emergence of an arriving primary user on that band. We refer to these two detection errors as class-A and class-B misdetection events, respectively. In this paper, we will only consider class-B misdetection events.

Misdetection events can negatively impact the performance of the system. When a misdetection event occurs, an ongoing SU may incorrectly detect that there is no PU arriving, but in fact, there is a PU entering the band. The PU will be blocked and the SU will be dropped into the retrial orbit at the same time. Meanwhile, a false alarm may also happen when an ongoing SU incorrectly detects the presence of a PU on the same channel, but in fact, there is no PU entering the channel. Once this occurs, the SU will be dropped into the retrial orbit. In this paper, we denote by $p_{m}$ and $p_{f}$ the probabilities of misdetection and false alarm, respectively.

Every arriving SU who wants to get service at the cognitive radio base station can decide whether or not to join the system. We will consider the unobservable case that SUs do not know the information (i.e., whether the PU band is available or not and the total SUs in the retrial orbit) about the system. After each service completed, an $\mathrm{SU}$ will get a reward of $R$ units. And the cost for delay in the system is charged by $C$ units pet time unit. All SUs want to maximize their own benefit and they are risk neutral. It is irrevocable for their decisions on joining or balking according to their assessment on the reward against the costs.

In the game-theoretic spectrum sharing model depicted in Fig. 1, we characterize SUs' strategies by a value $q \in$ $[0,1]$ which is the probability an SU decides to enter the system (thus, with probability $1-q$, the SU decides to leave the system), i.e., the effective entering probability for SUs is $\lambda_{s} q$. As all SUs are allowed to take their own decisions, this system can be regarded as a non-cooperative game and the aim of our investigation is to derive the symmetric Nash equilibria. We will study the SUs' equilibrium behavior and socially optimal strategies in the unobservable retrial queueing systems under the impact of sensing failures. Moreover, to use the PU band more efficiently and eliminate the difference between the equilibrium and the socially optimal strategies, we propose an effective approach of imposing an appropriate admission fee for SUs that decide to join the system. This control policy can induce individually optimizing SUs to behave in a socially optimal way and therefore to utilize the spectrum more economically.

For convenience, all notations used in this paper are listed in Table 1. For simplicity, denote by $\eta \equiv\left(1-p_{m}-p_{f}\right) \lambda_{p}$ and $\xi \equiv p_{m} \lambda_{p}+p_{f} \mu_{s}$, respectively. 


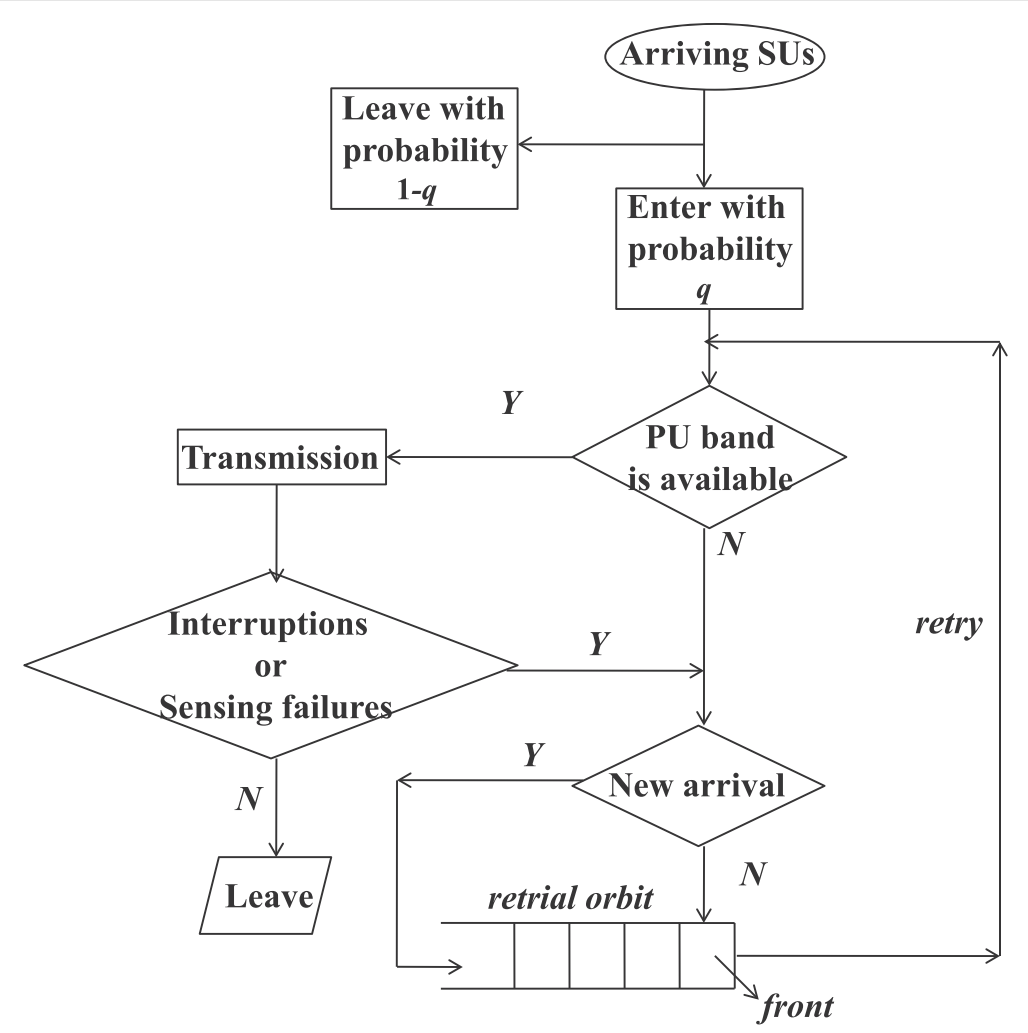

Fig. 1 Game-theoretic spectrum sharing model with imperfect sensing

\section{Equilibrium analysis and optimal control}

In this section, we first study the stability condition of this system and then give a game-theoretic equilibrium analysis. An optimal control policy is discussed based on the gap between equilibrium strategy and the socially optimal strategy of SUs.

\subsection{Stability condition and expected delay}

Let $(I(t), N(t))$ represent the state of the system at time

Table 1 Important notations

\begin{tabular}{ll}
\hline Symbol & Explanation \\
\hline$R$ & Reward for each service \\
$C$ & Cost per time unit \\
$\lambda_{s}$ & Arrival rate for primary SUs \\
$\lambda_{p}$ & Arrival rate for primary PUs \\
$\mu_{s}$ & Transmission rate for SUs \\
$\mu_{p}$ & Transmission rate for PUs \\
$\theta$ & Constant retrial rate \\
$p_{m}$ & The probability of misdetection \\
$p_{f}$ & The probability of false alarm \\
$p$ & Admission fee \\
\hline
\end{tabular}

$t$, where $I(t)$ denotes the state of the server (0, idle; 1 , serving an SU; 2 , serving a PU) and $N(t)$ records the number of the customers in the retrial orbit. From the model description, it is obvious that the process $\{I(t), N(t), t \geq$ $0\}$ is a continuous Markov chain with state space $\Omega=$ $\{(i, j), i=0,1,2, j \geq 0\}$. The system states and transition rate diagram are shown in Fig. 2.

Proposition 1. The quasi-birth-and-death (QBD) process $\{I(t), N(t)\}$ is positive recurrent if and only if the condition

$\left(\lambda_{s} q+\theta\right)\left[\mu_{p} \mu_{s}-\lambda_{s} q\left(\mu_{p}+\eta\right)\right]>\lambda_{s} q\left(\lambda_{p}+\mu_{p}\right)\left(\mu_{s}+\eta+\xi\right)$

is established.

Proof. The proof is given in Appendix 1.

Intuitively, the above condition enables the system not being too loaded and guarantees the existence of stationary distribution of the underlying Markov chain. Denote 


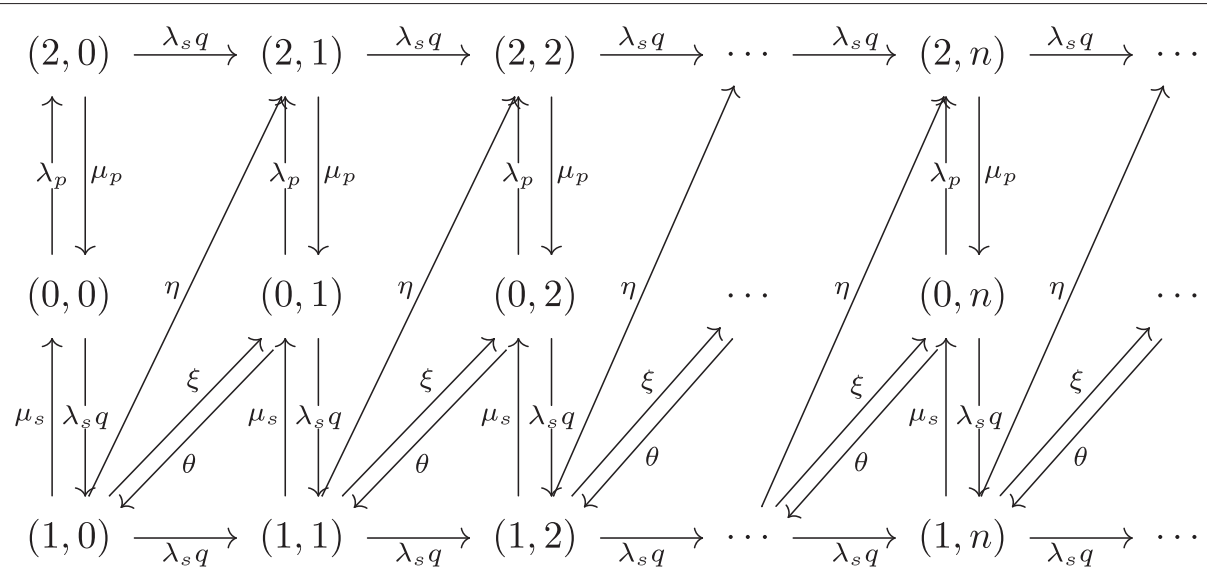

Fig. 2 Transition rate diagram in the cognitive radio system

by $p(i, j)$, the steady-state probability of state $(i, j)$ and the balance equations of the system is given below.

$$
\begin{aligned}
\left(\lambda_{p}+\lambda_{s} q\right) p(0,0)= & \mu_{p} p(2,0)+\mu_{s} p(1,0), \\
\left(\lambda_{p}+\lambda_{s} q+\theta\right) p(0, j)= & \mu_{p} p(2, j)+\mu_{s} p(1, j) \\
& +\xi p(1, j-1), j=1,2, \ldots \\
\left(\mu_{s}+\eta+\xi+\lambda_{s} q\right) p(1,0)= & \theta p(0,1)+\lambda_{s} q p(0,0) \\
\left(\mu_{s}+\eta+\xi+\lambda_{s} q\right) p(1, j)= & \theta p(0, j+1)+\lambda_{s} q p(1, j-1) \\
& +\lambda_{s} q p(0, j), j=1,2, \ldots \\
\left(\mu_{p}+\lambda_{s} q\right) p(2,0)= & \lambda_{p} p(0,0), \\
\left(\mu_{p}+\lambda_{s} q\right) p(2, j)= & \lambda_{p} p(0, j)+\lambda_{s} q p(2, j-1) \\
& +\eta p(1, j-1), j=1,2, \ldots
\end{aligned}
$$

We define the partial generating functions:

$$
p_{i}(z)=\sum_{j=0}^{\infty} z^{j} p(i, j), \quad i=0,1,2 .
$$

Multiplying Eqs. (1)-(6) by $z^{j}$ and summing up over $j$, we get the following equations.

$$
\begin{aligned}
\left(\lambda_{p}+\lambda_{s} q+\theta\right) p_{0}(z)-\theta p(0,0) & =\mu_{p} p_{2}(z)+\mu_{s} p_{1}(z) \\
+\xi z p_{1}(z) & \\
\left(\mu_{s}+\lambda_{s} q(1-z)+\eta+\xi\right) z p_{1}(z) & =\left(\lambda_{s} q z+\theta\right) p_{0}(z) \\
& -\theta p(0,0) \\
\left(\mu_{p}+\lambda_{s} q(1-z)\right) p_{2}(z) & =\eta z p_{1}(z)+\lambda_{p} p_{0}(z)
\end{aligned}
$$

After eliminating $p(0,0)$ from Eqs. (8) and (9) and combining with Eq. (10), we get

$$
\lambda_{s} q p_{0}(z)=\left(\mu_{s}-\lambda_{s} q z\right) p_{1}(z)-\lambda_{s} q p_{2}(z) .
$$

Inserting $z=1$ into Eqs. (10) and (11), we get the relations between $p_{0}(1), p_{1}(1)$ and $p_{2}(1)$ as follows:

$$
\begin{aligned}
& p_{2}(1)=\frac{\lambda_{p}\left(\mu_{s}-\lambda_{s} q\right)+\lambda_{s} q \eta}{\left(\lambda_{p}+\mu_{p}\right) \lambda_{s} q} p_{1}(1), \\
& p_{0}(1)=\frac{\mu_{p} \mu_{s}-\left(\mu_{p}+\eta\right) \lambda_{s} q}{\left(\lambda_{p}+\mu_{p}\right) \lambda_{s} q} p_{1}(1) .
\end{aligned}
$$

By virtue of the normalizing condition

$$
\sum_{j=0}^{\infty}(p(0, j)+p(1, j)+p(2, j))=p_{0}(1)+p_{1}(1)+p_{2}(1)=1
$$

we can get the probabilities that the PU band is idle, occupied by an SU, or occupied by a PU, respectively, given by

$$
\begin{aligned}
& p_{0}(1)=\frac{\mu_{p} \mu_{s}-\left(\mu_{p}+\eta\right) \lambda_{s} q}{\left(\lambda_{p}+\mu_{p}\right) \mu_{s}}, \\
& p_{1}(1)=\frac{\lambda_{s} q}{\mu_{s}}, \\
& p_{2}(1)=\frac{\lambda_{p}\left(\mu_{s}-\lambda_{s} q\right)+\lambda_{s} q \eta}{\left(\lambda_{p}+\mu_{p}\right) \mu_{s}} .
\end{aligned}
$$

The expected number of customers in the retrial orbit under the state $i$ is therefore given by

$$
E\left[R_{i}\right]=\sum_{j=0}^{\infty} j p(i, j), i=0,1,2
$$

With the help of $p_{i}(z)$, we obtain that

$$
E\left[R_{i}\right]=p_{i}^{\prime}(z) \mid z=1 .
$$


Differentiating Eqs. (8), (10), and (11), and taking $z=1$ yields

$$
\begin{aligned}
\left(\lambda_{p}+\lambda_{s} q+\theta\right) p_{0}^{\prime}(1)= & \xi\left(p_{1}(1)+p_{1}^{\prime}(1)\right)+\mu_{p} p_{2}^{\prime}(1) \\
& +\mu_{s} p_{1}^{\prime}(1), \\
-\lambda_{s} q p_{2}(1)+\mu_{p} p_{2}^{\prime}(1)= & \eta\left(p_{1}(1)+p_{1}^{\prime}(1)\right)+\lambda_{p} p_{0}^{\prime}(1), \\
\lambda_{s} q p_{0}^{\prime}(1)+\lambda_{s} q p_{1}(1)= & \left(\mu_{s}-\lambda_{s} q\right) p_{1}^{\prime}(1)-\lambda_{s} q p_{2}^{\prime}(1) .
\end{aligned}
$$

From Eqs. (15)-(17), we can easily get the expressions of $p_{0}^{\prime}(1), p_{1}^{\prime}(1)$ and $p_{2}^{\prime}(1)$. Hence, the expected number of customers in the system is given by

$$
\begin{aligned}
N= & \sum_{i=0}^{i=2} E\left[R_{i}\right]+p_{1}(1) \\
= & \frac{\lambda_{s} q\left(\lambda_{s} q+\theta\right)\left[\left(\lambda_{p}+\mu_{p}\right)\left(\mu_{p}+\eta\right)+\lambda_{p}\left(\mu_{s}-\lambda_{s} q\right)+\lambda_{s} q \eta\right]}{\left(\lambda_{p}+\mu_{p}\right)\left\{\left(\lambda_{s} q+\theta\right)\left[\mu_{p} \mu_{s}-\lambda_{s} q\left(\mu_{p}+\eta\right)\right]-\lambda_{s} q\left(\lambda_{p}+\mu_{p}\right)\left(\mu_{s}+\eta+\xi\right)\right\}} \\
& +\frac{\lambda_{s} q\left[\lambda_{p}\left(\mu_{s}-\lambda_{s} q\right)+\lambda_{s} q \eta+\left(\lambda_{p}+\mu_{p}\right)(\eta+\xi)\right]}{\left(\lambda_{s} q+\theta\right)\left[\mu_{p} \mu_{s}-\lambda_{s} q\left(\mu_{p}+\eta\right)\right]-\lambda_{s} q\left(\lambda_{p}+\mu_{p}\right)\left(\mu_{s}+\eta+\xi\right)} .
\end{aligned}
$$

Further, the expected delay of an arriving SU is given by

$$
T(q)=\frac{N}{\lambda_{s} q} .
$$

\subsection{Nash equilibrium}

Based on the results obtained above, the equilibrium behavior of SUs is given as follows.

Theorem 1. In the considered model, a unique mixed equilibrium strategy which is the joining probability $q_{e}$ is given by

$$
q_{e}= \begin{cases}0, & \text { if } R \leq C T(0), \\ q_{e}, & \text { if } C T(0)<R<C T(1), \\ 1, & R \geq C T(1),\end{cases}
$$

where $q_{e}{ }^{*}$ satisfies the equation $C T\left(q_{e}{ }^{*}\right)=R$.

Proof. The proof is presented in Appendix 2.

Remark 1. Suppose that $q$ is the joining probability of other arriving SUs, if $q<q_{e}$, we can conclude that the expected net benefit of the tagged SU is positive once he enters the system. In this case, the unique response is 1 . Similarly, the unique best response is 0 if $q>q_{e}$. What is more, any strategy between 0 and 1 is a best response if $q=q_{e}$. This shows that an individual's best response is an decreasing function of the strategy by the others, i.e., the higher the joining probability selected by the others, the lower is one's best response. Therefore, we have an "avoid the crowd" (ATC) situation. We conclude that $q_{e}$ is the unique equilibrium strategy.

\subsection{Socially optimal strategy}

Now, we turn our attention to social optimization. In the real situation where resources are limited, this queueing system considered from a social point of view is of great significance. The social objective function is defined as

$$
S_{s o c}=\lambda_{s} q(R-C T(q)),
$$

where $\lambda_{s} q$ is the effective arrival rate. Let $q_{s o c}$ be the optimal joining strategy. By solving

$$
q^{*}=\arg \max _{0 \leq q \leq 1}\left\{\lambda_{s} q(R-C T(q))\right\},
$$

we can get the following results.

Theorem 2. In the considered model, a unique socially optimal joining probability $q_{s o c}$ adopted by the SUs which can be expressed as

$$
q_{s o c}= \begin{cases}0, & q^{*} \leq 0 \\ q^{*}, & \text { if } 0<q^{*}<1, \\ 1, & \text { if } q^{*} \geq 1\end{cases}
$$

Proof. Since $T(q)$ is increasing with $q$, the function to be maximized is strictly concave and has a unique maximum $q^{*}$.

We can infer $q^{*} \leq q_{e}$ due to $\left.\frac{d\left(S_{s o c}\right)}{d q}\right|_{q=q_{e}}=\lambda_{s}(R-$ $\left.C T\left(q_{e}\right)\right)-\left.\lambda_{s} q_{e} C \frac{d T(q)}{d q}\right|_{q=q_{e}} \leq 0$. It shows that individual optimization leads to a longer queue than the desired socially optimal strategy. We can impose an appropriate admission fee on the SUs who enter the system to gap this difference.

\subsection{Admission fee}

We have derived the equilibrium strategy and the social optimization strategy of SUs upon arrival. It is easy to see that these two strategies do not coincide with each other, and the relationship $q^{*} \leq q_{e}$ holds. From the managerial point of view, this leads to the fact that the limited resources will be used excessively, as all users want to maximize their own benefit regardless of others. In order to reduce the gap between individual and social optimization and let SUs behave in a socially optimal way, the administrator of the cognitive radio base station is likely to impose a constant admission fee $p$ on SUs when they decide to enter the system.

When the admission fee $p$ is imposed, the reward for an SU who enters the system is reduced into $R-p$. As the administrator absorbs every SU's surplus, then the equilibrium joining strategy $q_{e}(p)$ is changed into
1) $R-p \leq C T(0): q_{e}(p)=0$;
2) $C T(0)<R-p<C T(1): q_{e}(p)$ satisfies the equation $C T\left(q_{e}(p)\right)=R-p$
3) $R-p \geq C T(1): q_{e}(p)=1$. 
Under the condition of being imposed an admission fee, the social benefit of the system is $\lambda q[R-p-$ $C E[T(\lambda)]]+\lambda q p$ which equals to $S_{s o c}$. Note that as the admission fee $p$ has no effect on the social objective function, the final socially optimal joining strategy will not be changed. To eliminate the difference between the equilibrium joining strategy and the socially optimal solution, an optimal admission fee $p^{*}$ should satisfy the equation $q_{s o c}=q_{e}\left(p^{*}\right)$.

\section{Numerical examples}

In this section, we focus on the effects of different parameters on the behavior of SUs via numerical examples. More concretely, we first examine how the equilibrium and socially optimal entrance probabilities are affected by changing the values of parameters $\lambda, \mu, \eta, \beta, \theta$, and $R$. It is not hard to find that $q^{*}$ is smaller than $q_{e}$ in all these figures, as explained before. The impact of misdetections on equilibrium and socially optimal behavior can also be observed.

It is shown in Fig. 3 that both $q_{e}$ and $q^{*}$ are decreasing as the arrival rate $\lambda_{s}$ increases. This is because when $\lambda_{s}$ increases, arriving SUs who do not know whether the PU band is available or not will see more blocked SUs waiting in the retrial orbit. So the arriving SUs are less inclined to join the orbit to avoid more waiting cost as they are not allowed to balk during their waiting. Figure 4 depicts the influence of service rate $\mu_{s}$ on the strategic entrance probabilities. We observe that $q_{e}$ and $q^{*}$ are increasing with respect to $\mu_{s}$. It can be explained that the increasing service rate of PU band benefits SUs waiting in the retrial orbit as the completing service time for SUs get faster. As in Fig. 5, the strategic entrance probabilities decreases as the arrival rate $\lambda_{p}$ of PUs increases. This is due to the priority of PUs, and when $\lambda_{p}$ increases, the interruption times per unit become more frequent. The server needs some time to serve the PU. So SUs are reluctant to join the orbit upon arrival. The system will get more loaded as the server's breakdown become more frequent. Considering the influence of $\mu_{p}$ on these two entrance probabilities, we observe in Fig. 6 that along with the increasing of $\mu_{p}$, the expected sojourn time for an PU becomes shorter. There will be more opportunities for arriving SUs who stay in the orbit to use the PU band. When it comes to $\theta$ in Fig. 7, PUs are more willing to join the system in pace with the increasing retrial rate of the SUs in orbit. When $\theta$ increases, SUs will have more probabilities to get successful to retry for using the PU band during the same period. All the above figures show that the equilibrium strategies of SU are larger than the socially optimal strategy, so it is of significance to impose an admission on the administrator of the network. As for the impact of the probabilities of misdetection and false alarm on the behavior of SUs, it can be seen from Fig. 8 that arriving SUs will be more likely to enter the system as $p_{m}$ increases and the phenomenon is reverse as $p_{f}$ increases. Because the arriving PU will be forced to drop when misdetection occurs, the SUs' waiting time in the retrial orbit will be reduced. However, if $p_{f}$ increases, the $\mathrm{SU}$ who is in service tends to give up and enters the retrial orbit, which incurs negative externalities on those who stay in the retrial orbit. The same phenomenon is observed for the maximum social strategies as shown in Fig. 9. It is interesting to see that in Fig. 10, the arriving SUs will be imposed more fees as $p_{m}$ increases or $p_{f}$ decreases, because the amount of SUs in the system will increase in both cases and the negative externalities lead to these results.

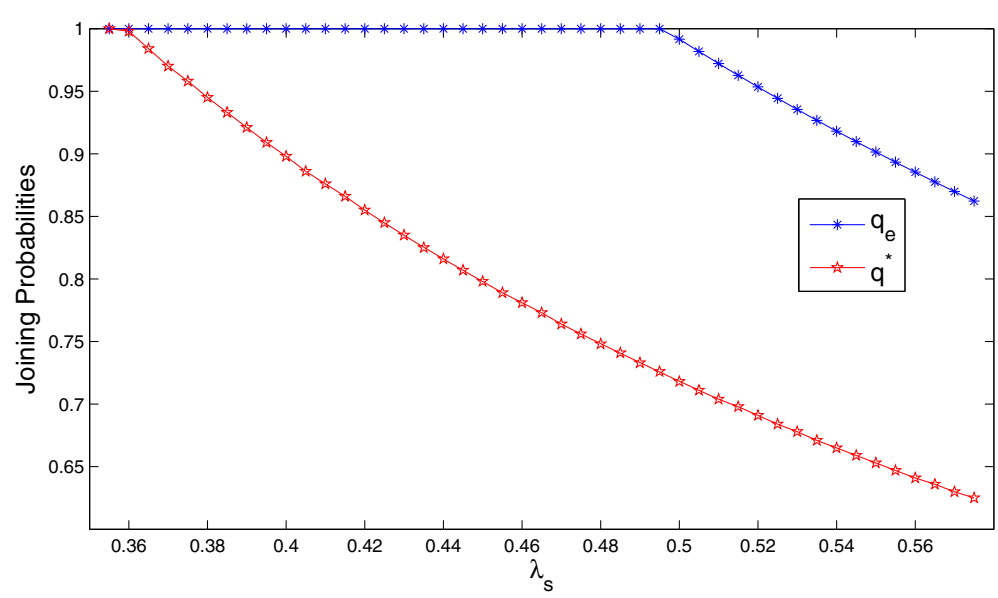

Fig. 3 Equilibrium and social optimization joining probabilities vs. $\lambda_{s}$ for $R=10, C=1, \mu_{s}=2, \lambda_{p}=0.4, \mu_{p}=2, \theta=0.7, p_{m}=0.001$, and $p_{f}=0.001$ 


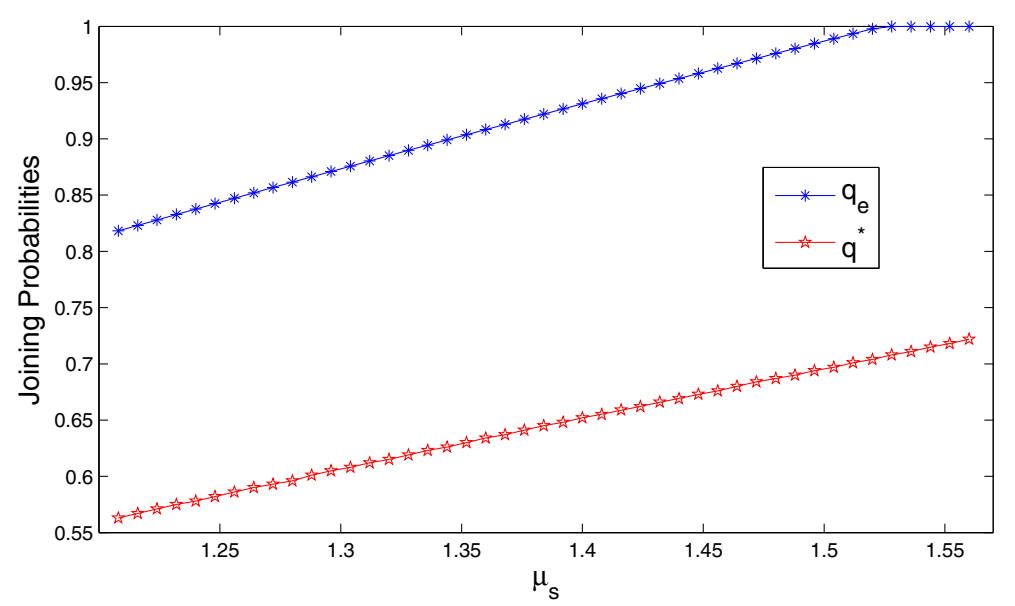

Fig. 4 Equilibrium and social optimization joining probabilities vs. $\mu_{s}$ for $R=10, C=1, \lambda_{s}=0.4, \lambda_{p}=0.4, \mu_{p}=2, \theta=0.7, p_{m}=0.001$, and $p_{f}=0.001$

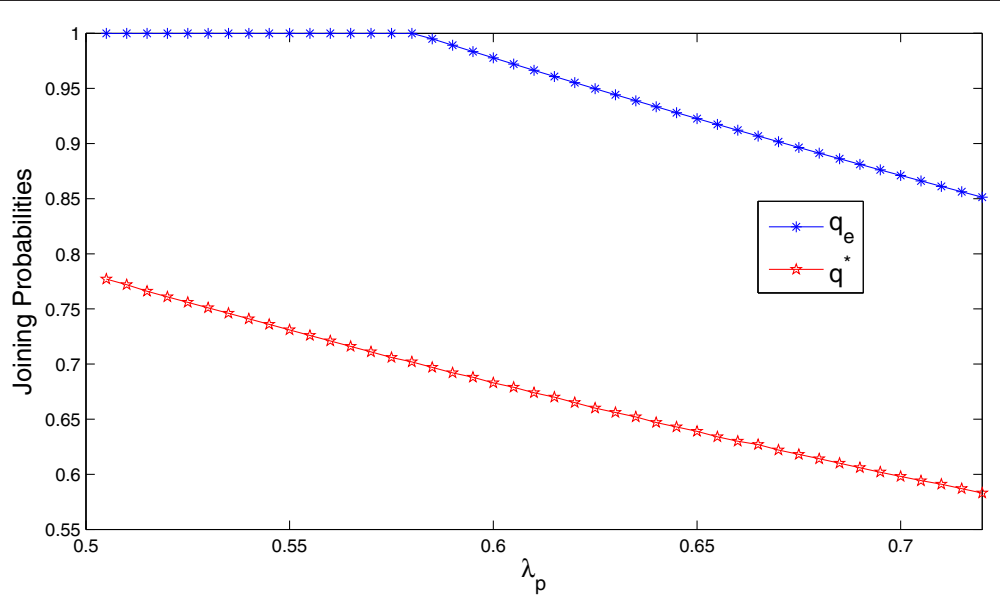

Fig. 5 Equilibrium and social optimization joining probabilities vs. $\lambda_{p}$ for $R=10, C=1, \mu_{s}=2, \lambda_{s}=0.4, \mu_{p}=2, \theta=0.7, p_{m}=0.001$, and $p_{f}=0.001$

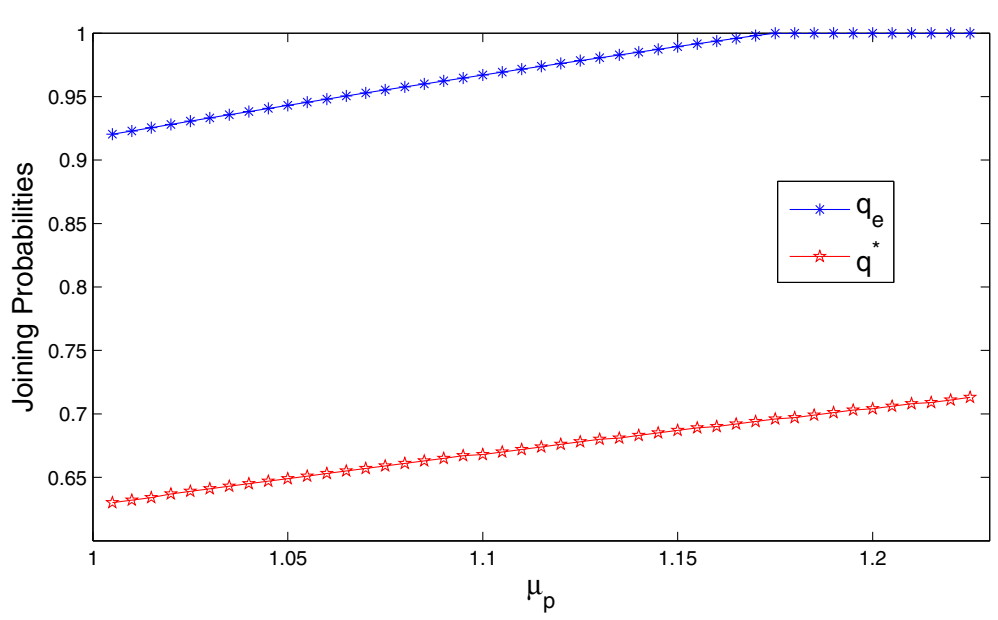

Fig. 6 Equilibrium and social optimization joining probabilities vs. $\mu_{p}$ for $R=10, C=1, \mu_{s}=2, \lambda_{p}=0.4, \lambda_{s}=0.4, \theta=0.7, p_{m}=0.01$, and $p_{f}=0.01$ 


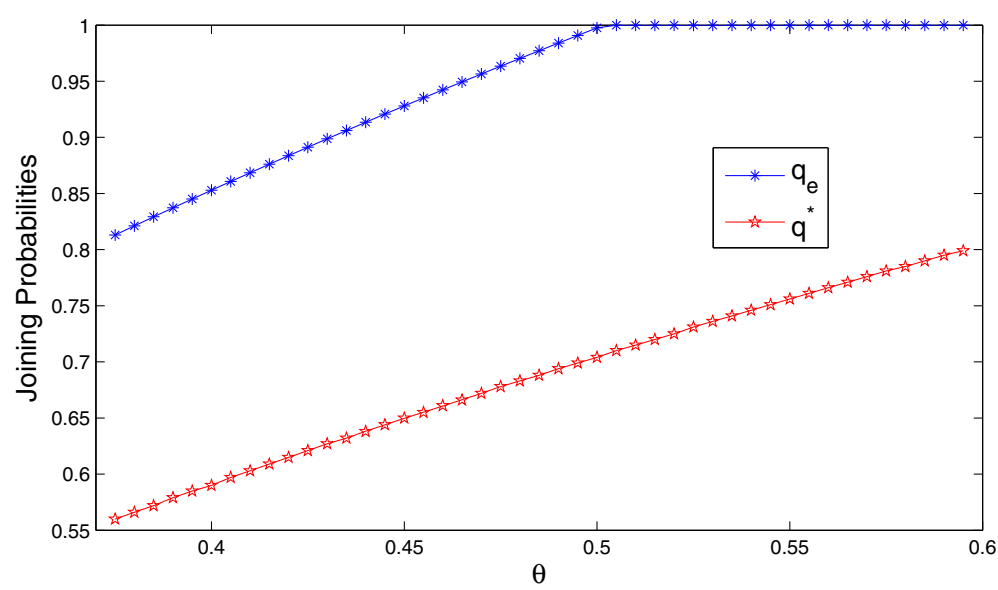

Fig. 7 Equilibrium and social optimization joining probabilities vs. $\theta$ for $R=10, C=1, \mu_{s}=2, \lambda_{s}=0.4, \lambda_{p}=0.4, \mu_{p}=2, p_{m}=0.01$, and $p_{f}=0.01$

\section{Conclusions}

In this paper, we considered the SUs' joining behavior in cognitive radio network with a single bandwidth under imperfect spectrum sensing. We used the constant retrial queueing system with server breakdowns to model the actual situations in which the PUs own priority over SUs and SUs will retry their luck for service if interrupted or blocked upon arrival. The SUs' joining behavior were described from an economic viewpoint based on gametheoretic analysis. The equilibrium and socially optimal strategies of SUs were investigated. It was shown that the equilibrium strategy is greater than the socially optimal strategy, and it was verified through numerical examples.
To eliminate the gap between equilibrium strategy and socially optimal strategy, we proposed a control policy that imposes an admission fee on each joining SU in order to utilize the PU band more efficiently.

\section{Appendix 1: Proof of Proposition 1}

Proof. Using the lexicographical sequence for the states, the infinitesimal generator $Q$ can be written as

$$
Q=\left(\begin{array}{ccccccc}
A_{0} & C & & & & \\
B & A & C & & & \\
& B & A & C & & \\
& & & \ddots & \ddots & \ddots
\end{array}\right),
$$

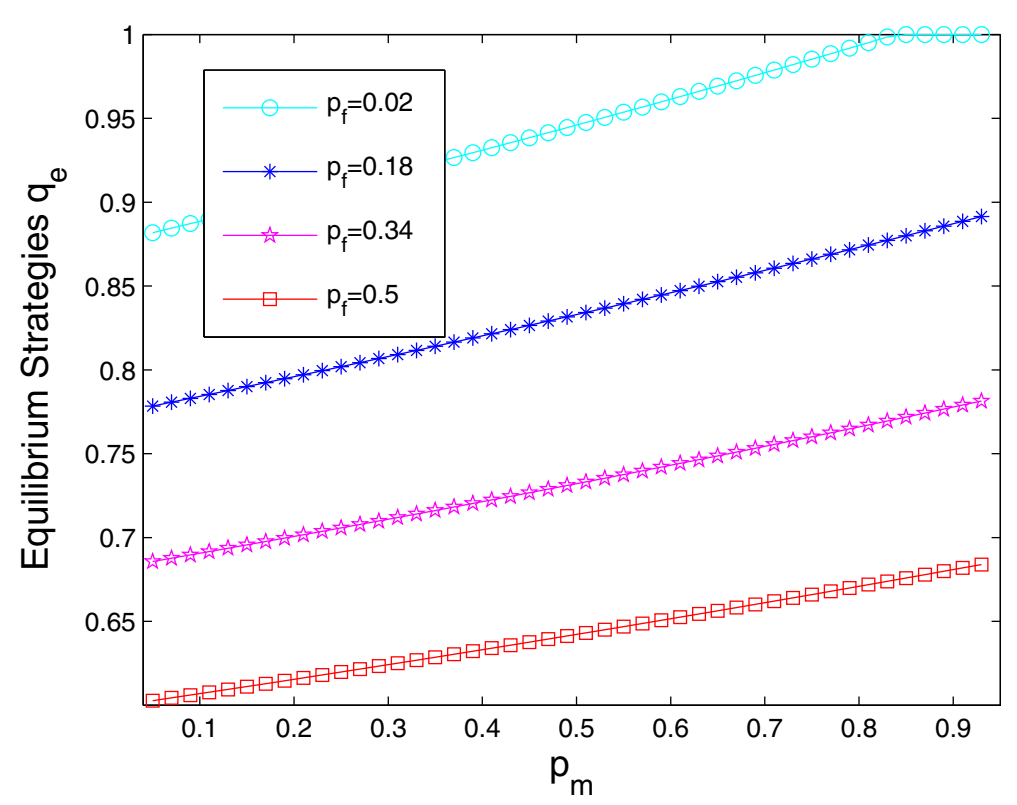

Fig. 8 Equilibrium strategies vs. $p_{m}$ for $R=15, C=3, \mu_{s}=2, \lambda_{s}=0.4, \lambda_{p}=0.5, \mu_{p}=2$, and $\theta=0.7$ 


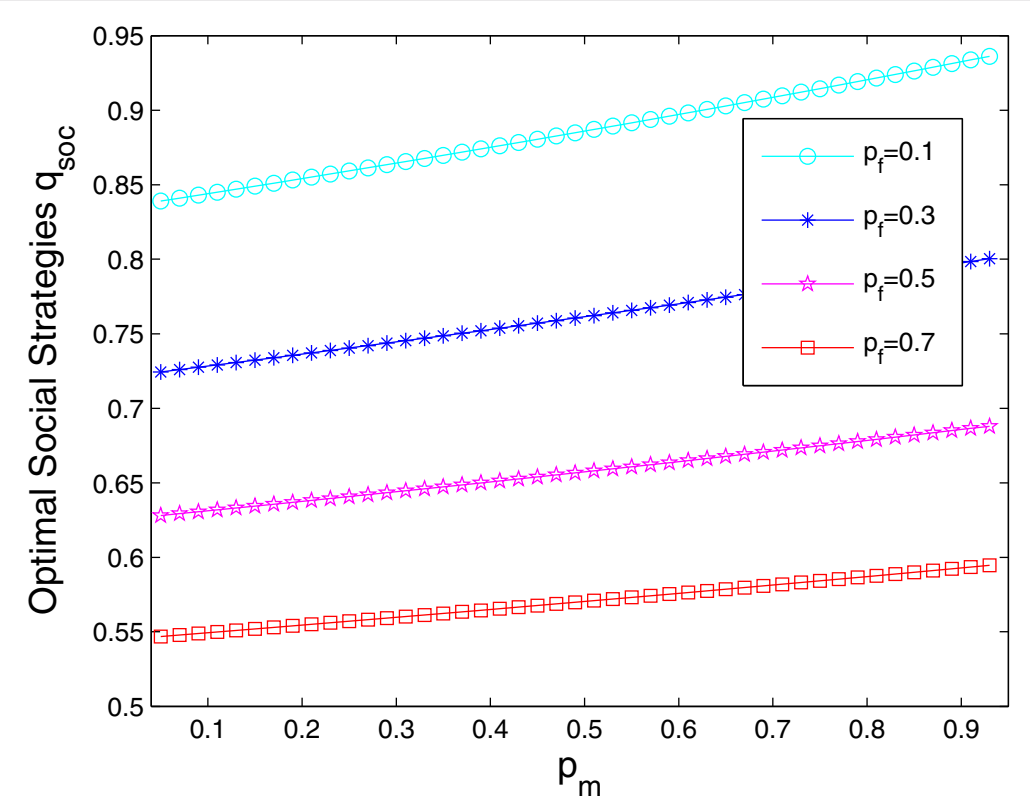

Fig. 9 Optimal social strategies vs. $p_{m}$ for $R=10, C=1, \mu_{s}=2, \lambda_{s}=0.4, \lambda_{p}=0.4, \mu_{p}=2$, and $\theta=0.7$

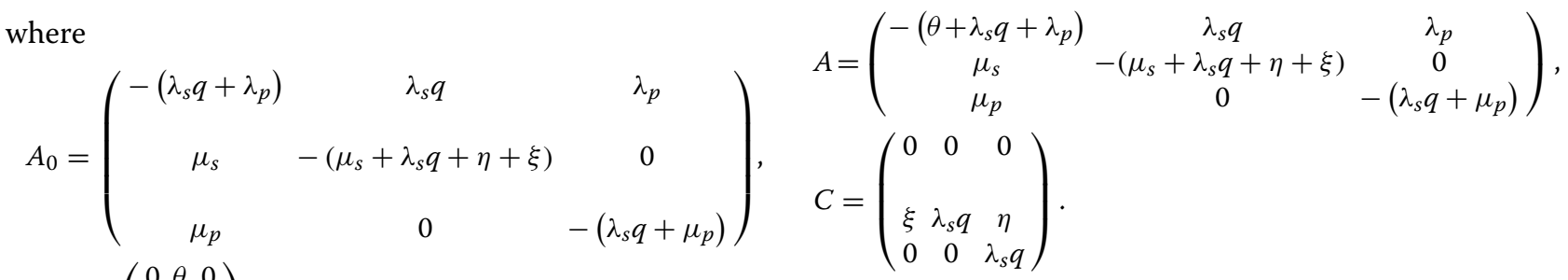
$B=\left(\begin{array}{lll}0 & \theta & 0 \\ 0 & 0 & 0 \\ 0 & 0 & 0\end{array}\right)$,

Due to the block structure of matrix $Q,\{I(t), N(t)\}$ is called a quasi-birth-and-death (QBD) process.

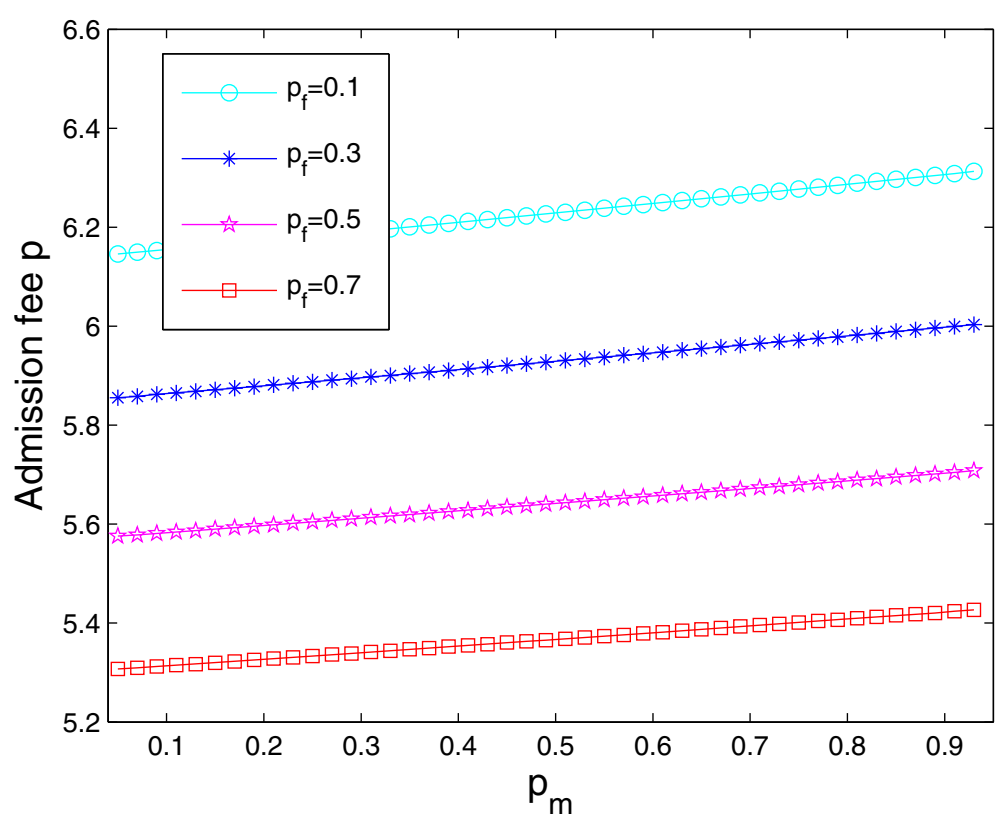

Fig. 10 Admission fee vs. $p_{m}$ for $R=10, C=1, \mu_{s}=2, \lambda_{s}=0.4, \lambda_{p}=0.4, \mu_{p}=2$, and $\theta=0.7$ 
First, we assume

$D=B+A+C=\left(\begin{array}{ccc}-\left(\theta+\lambda_{s} q+\lambda_{p}\right) & \theta+\lambda_{s} q & \lambda_{p} \\ \mu_{s}+\xi & -\left(\mu_{s}+\eta+\xi\right) & \eta \\ \mu_{p} & 0 & -\mu_{p}\end{array}\right)$.

Since $D$ is reducible, the Theorem 7.3.1 in [19] gives the condition for positive recurrence of the QBD. After permutation of rows and columns, the Theorem 7.3.1 states that the QBD is positive recurrent if and only if

$$
\boldsymbol{v}\left(\begin{array}{lll}
0 & \theta & 0 \\
0 & 0 & 0 \\
0 & 0 & 0
\end{array}\right) \mathbf{1}>\boldsymbol{v}\left(\begin{array}{ccc}
0 & 0 & 0 \\
\xi & \lambda_{s} q & \eta \\
0 & 0 & \lambda_{s} q
\end{array}\right) \mathbf{1}
$$

where $\mathbf{1}$ is a column vector with all elements equal to one, and $\boldsymbol{v}$ is the unique solution $\boldsymbol{v} D=\mathbf{0 , v} \mathbf{1}=1$. After some algebraic manipulation, the QBD process is positive recurrent if and only if

$$
\left(\lambda_{s} q+\theta\right)\left[\mu_{p} \mu_{s}-\lambda_{s} q\left(\mu_{p}+\eta\right)\right]>\lambda_{s} q\left(\lambda_{p}+\mu_{p}\right)\left(\mu_{s}+\eta+\xi\right)
$$

are established. The right side of the inequality is always greater than zero which infers to

$$
\mu_{p} \mu_{s}>\lambda_{s} q\left(\mu_{p}+\eta\right)
$$

Therefore, the Eqs. from (12) to (14) are greater than zero which is reasonable.

\section{Appendix 2: Proof of Theorem 1}

Proof. The expected waiting time of the tagged SU who choose to enter system is increasing with the same strategies $q$ adopted by other SUs. We can output the expected waiting time as in (25).

$$
\begin{aligned}
& T(q) \\
& =\frac{\left(\lambda_{s} q+\theta\right)\left[\left(\lambda_{p}+\mu_{p}\right)\left(\mu_{p}+\eta\right)+\lambda_{p}\left(\mu_{s}-\lambda_{s} q\right)+\lambda_{s} q \eta\right]}{\left(\lambda_{p}+\mu_{p}\right)\left\{\left(\lambda_{s} q+\theta\right)\left[\mu_{p} \mu_{s}-\lambda_{s} q\left(\mu_{p}+\eta\right)\right]-\lambda_{s} q\left(\lambda_{p}+\mu_{p}\right)\left(\mu_{s}+\eta+\xi\right)\right\}} \\
& +\frac{\lambda_{p}\left(\mu_{s}-\lambda_{s} q\right)+\lambda_{s} q \eta+\left(\lambda_{p}+\mu_{p}\right)(\eta+\xi)}{\left(\lambda_{s} q+\theta\right)\left[\mu_{p} \mu_{s}-\lambda_{s} q\left(\mu_{p}+\eta\right)\right]-\lambda_{s} q\left(\lambda_{p}+\mu_{p}\right)\left(\mu_{s}+\eta+\xi\right)} .
\end{aligned}
$$

Differentiating the denominator in the second fraction (denoted as $g(q))$ of $T$ we can obtain

$$
\begin{aligned}
g^{\prime}(q)= & -\lambda_{s}\left(2 \lambda_{s} q+\theta\right)\left(\mu_{p}+\eta\right)-\lambda_{s} \lambda_{p}\left(\mu_{s}+\eta+\xi\right) \\
& -\lambda_{s} \mu_{p}(\eta+\xi)<0 .
\end{aligned}
$$

Thus, it is decreasing with $q$. Observing the whole expression of $T$, it is mean to identify the monotonicity of $\frac{\lambda_{p}\left(\mu_{s}-\lambda_{s} q\right)+\lambda_{s} q \eta}{g(q)}$. It is easier to prove the inverse faction which is $\frac{g(q)}{\lambda_{p}\left(\mu_{s}-\lambda_{s} q\right)+\lambda_{s} q \eta}$ is monotone decreasing as $q$ increases. Just differentiate the objective function, and we omitted it.
Therefore, the payoff for the tagged SU who chooses to enter the system (means that the tagged SU selects the strategy 1 ) when all others select strategy $q$ is

$$
S(q)=R-C T(q)
$$

For all the arriving SUs, each has two pure strategies: to join or balk and a mixed strategy. We denote these pure and mixed strategies by a fraction $q, 0 \leq q \leq 1$. So the mixed strategy means an SU enters the system with probability $q$ and not to join with probability $1-q$. Let $q_{e}$ be the individual equilibrium strategy of each $\mathrm{SU}$, then we analyze the equilibrium behavior of arriving SUs as three cases below:

1) $R \leq C T(0)$. An $\mathrm{SU}$ who joins can get a negative benefit when there are no other SUs entering the system. So his decision is not to join. Therefore, the strategy of joining with probability $q_{e}=0$ is an equilibrium strategy.

2) $C T(0)<R<C T(1)$. We can specify that if $q_{e}=1$, then an SU who joins gets a negative benefit. So this is not an equilibrium strategy. If $q_{e}=0$, an SU who joins obtains a positive profit which is more than by balking (the benefit is 0 ). Thus, this is not an equilibrium strategy too. Therefore, there exists a unique equilibrium strategy $q_{e}$ such that $C T\left(q_{e}\right)=R$.

3) $R \geq C T(1)$. In this case, any arriving SU will obtain a non-negative profit even if all other SUs join the system. So, the only one equilibrium strategy of joining the system is $q_{e}=1$. And joining is a dominant strategy.

\section{Competing interests}

The authors declare that they have no competing interests.

\section{Acknowledgments}

This work was supported by the National Natural Science Foundation of China (Grant Nos. 11171019, 71390334 and 71571014), the 111 Project of China (B16002) and US National Science Foundation (Grant Nos. 1137732 and 1241626)

\section{Author details}

${ }^{1}$ Department of Mathematics, Beijing Jiaotong University, No. 3 Shang Yuan Cun, Beijing, China. ${ }^{2}$ Department of Computer Science and the NSF Center for Research on Complex Networks, Texas Southern University, Houston, TX 77004, Houston, USA.

Received: 30 December 2015 Accepted: 14 May 2016

Published online: 02 June 2016

\section{References}

1. J Mitola III, Cognitive radio for flexible mobile multimedia communications. Mobile Netw. Appl. 6, 435-441 (2001)

2. Q Zhao, BM Sadler, A survey of dynamic spectrum access. IEEE Signal Process. Mag. 24, 79-89 (2007)

3. NH Tran, CS Hong, Z Han, S Lee, Optimal pricing effect on equilibrium behaviors of delay-sensitive users in cognitive radio networks. Selected Areas in Commun. IEEE J. 31(11), 2566-2579 (2013)

4. CT Do, NH Tran, Z Han, LB Le, S Lee, CS Hong, Optimal pricing for duopoly in cognitive radio networks: cooperate or not cooperate? Wireless Commun. IEEE Trans. 13(5), 2574-2587 (2014) 
5. S Lee, H Choi, CK Kim, in 9th International Symposium on Communications and Information Technology (ISCIT 2009). A game-theoretic analysis of spectrum sharing in cognitive radio networks, vol. 9, (2009), pp. 415-416

6. A Garhwal, PP Bhattacharya, A study on dynamic spectrum access techniques for cognitive radio. Intl. J. Next-Generation Netw. (IJNGN). 3, 15-32 (2011)

7. J Elias, F Martignon, A Capone, E Altman, Non-cooperative spectrum access in cognitive radio networks: a game theoretical model. Comput. Netw. 55, 3832-3846 (2011)

8. H Li, Z Han, Socially optimal queuing control in CR networks subject to service interruptions: to queue or not to queue? IEEE Trans. Wireless Commun. 10, 1656-1666 (2011)

9. CT Do, NH Tran, M Van Nguyen, S Lee, Social optimization strategy in unobserved queueing systems in cognitive radio networks. IEEE Commun. Lett. 16, 1944-1947 (2012)

10. K Jagannathan, I Menache, E Modiano, G Zussman, Non-cooperative spectrum access-the dedicated vs. free spectrum choice. IEEE J. Selected Areas Commun. 30, 2251-2261 (2012)

11. J Wang, W Li, Non-cooperative and cooperative joining strategies in cognitive radio networks with random access. IEEE Trans. Veh. Technol. doi:10.1109/TVT.2015.2470115

12. AT Hoang, Y-C Liang, DTC Wong, Y Zeng, R Zhang, Opportunistic spectrum access for energy-constrained cognitive radios, IEEE Trans, Wireless Commun. 8(3), 1206-1211 (2009)

13. I Suliman, J Lehtomaki, T Braysy, K Umebayashi, in Proc. IEEE Personal Indoor and Mobile Radio Communications. Analysis of cognitive radio networks with imperfect sensing, (2009), pp. 1616-1620

14. X Gelabert, O Sallent, JP Romero, R Agusti, Spectrum sharing in cognitive radio networks with imperfect sensing: a discrete-time Markov model. Comput. Netw. 54, 2519-2536 (2010)

15. STang, BL Mark, Modeling and analysis of opportunistic spectrum sharing with unreliable spectrum sensing. IEEE Trans. Wireless Commun. 8, 1934-1943 (2009)

16. T Ngatched, S Dong, AS Alfa, Analysis of cognitive radio networks with channel assembling, buffering, and imperfect sensing. Wireless Commun. Netw. Conf. (WCNC). 2013, 952-957 (2013)

17. AE Shafie, Optimal spectrum access for cognitive radios, arXiv preprint, arXiv:1208.4508 [cs.IT] (2012). http://arxiv.org/abs/1208.4508

18. O Altrad, S Muhaidat, A Al-Dweik, Opportunistic spectrum access in cognitive radio networks under imperfect spectrum sensing. IEEE Trans. Veh. Technol. 63, 920-925 (2014)

19. G Latouche, V Ramaswami, Introduction to matrix analytic methods in stochastic modeling. ASA-SIAM Series on Statistics and Applied Probability, Philadelphia, Pennsylvania (1999)

\section{Submit your manuscript to a SpringerOpen ${ }^{\circ}$ journal and benefit from:}

- Convenient online submission

- Rigorous peer review

- Immediate publication on acceptance

- Open access: articles freely available online

- High visibility within the field

- Retaining the copyright to your article

Submit your next manuscript at $\gg$ springeropen.com 